\title{
LAS PRIMERAS ANGIOSPERMAS EN EL CRETÁCICO DE LA CUENCA NEUQUINA (CENTRO OESTE DE ARGENTINA): ASPECTOS GEOLÓGICOS RELACIONADOS
}

\author{
PATRICIA SILVINA VALLATI \\ Laboratorio de Bioestratigrafía, UNPSJB, Ciudad Universitaria, 9000, Comodoro Rivadavia, Chubut, Argentina. \\ patricia.vallati@gmail.com
}

\begin{abstract}
RESUMO - Apresenta-se o registro fóssil das primeiras palinofloras cretácicas que inclui grãos de angiospermas na Bacia Neuquina (Patagônia setentrional da Argentina), destacando seu marco estratigráfico-geotectônico. O primeiro registro de angiospermas da Bacia Neuquina ocorre na Seção litológica K do Grupo Huitrín-Rayoso (Zona Afropollis zonatus, de idade aptiana). Nesta palinoflora aparecem grãos de angiospermas com aberturas primitivas (monossulcados) junto com outros de tipos aperturais relativamente mais complexos (pentaoctocotomossulcados e com abertura zonissulcada-operculada). A associação inclui Afropollis, Asteropollis, Clavatipollenites e Retimonocolpites. Os níveis férteis subjacentes ao grupo mencionado, não registraram, até o momento, uma associação com grãos de angiospermas basais (monosulcados, tectados, columelados), como a que está presente no Barremiano de diferentes partes do mundo. O evento neuquino de aparecimento das angiospermas, com um provável hiato bioestratigráfico basal, está relacionado às mudanças tectônicas do Cretáceo médio inicial (movimentos mirânicos iniciais) e, conseqüentemente, à ruptura do isolamento geográfico da biota que prevaleceu no Cretáceo inicial. O próximo passo na diversificação das angiospermas neuquinas foi evidenciado na Formação Huincul, uma das unidades basais dos "Estratos com Dinossauros", na localidade de El Zampal. A Zona Fraxinoipollenites fragilis (Albiano?/Cenomaniano) inclui as primeiras eudicotiledôneas. É possível que este registro esteja relacionado com as modificações fisiográficas vinculadas à Orogenia Mirânica principal.
\end{abstract}

Palavras-chave: Angiospermas, pólen, Cretáceo médio, geotectônica.

\begin{abstract}
FIRST ANGIOSPERMS IN THE CRETACEOUS OF THE NEUQUÉN BASIN (WESTCENTRAL ARGENTINA): RELATED GEOLOGICAL ASPECTS. The first record of Cretaceous palynofloral assemblages including angiosperm pollen grains in the Neuquén Basin (septentrional Patagonia of Argentina) is here considered outlining its stratigraphic-geotectonic frame. In the Neuquén Basin, the first angiosperm pollen grains are recorded in the lithologic Section K of the Huitrín-Rayoso Group (Afropollis zonatus Zone of Aptian age). This palynoflora includes angiosperm pollen grains with a primitive aperture (monosulcate grains) as well as grains with relatively more advanced apertural types (penta-octochotomosulcate and zonisulcate-operculate apertures). Afropollis, Asteropollis, Clavatipollenites and Retimonocolpites are recorded. Up to now, a basal angiosperm assemblage including monosulcate grains was not recognized in the lower fertile levels of the mentioned group. The same is present in the Barremian of several localities around the world. The Neuquean event of angiosperm appearance and the suggested basal biostratigraphic "hiatus" is related to the early middle Cretaceous tectonic activity (initial miranic movements), and consequently to the ending of the geographic isolation of the biota which prevailed during the early Cretaceous. The next stage of the Neuquean angiosperm diversification is recognized in the Huincul Formation, one of the basal units of the "Dinosaurian Beds", in the El Zampal locality. The first eudicotiledonean grains are recorded in the Fraxinoipollenites fragilis Zone (Albian?/Cenomanian). This zone includes tricolpate grains as well as grains with diversified apertural types (tricolporoidate and tricolporate grains). This record can possibly be related to the principal Miranic Orogeny fisiographic changes.
\end{abstract}

Key words: Angiosperm, pollen, middle Cretaceous, geotectonic.

\section{INTRODUCCIÓN Y ASPECTOS GEOLÓGICOS}

Las palinofloras cretácicas a las que se hace referencia en este trabajo, proceden de la Comarca Bajada del Agrio (Bajada del Agrio, Agrio del Medio y km 803) en la Provincia del Neuquén y de la localidad El Zampal, sur de la Provincia de Mendoza, en la Cuenca Neuquina (Figura 1). Los depósitos predominantemente continentales del Grupo Huitrín-Rayoso (Grupo H-R) y de la base de los "Estratos con Dinosaurios" [= Grupo Pehuenche Doering, no Grupo Neuquén (nombre 
inválido); cf. Groeber (1959)] suprayacen niveles marinos con amonites. En particular, la zona eobarremiana de Paraspiticeras groeberi Aguirre Urreta \& Rawson (1993), reconocida en los términos más altos de la Formación Agrio (Grupo Mendoza), permite establecer un límite cronológico inferior confiable para datar en forma indirecta las palinofloras en las unidadaes suprayacentes.

\section{Grupo Huitrín-Rayoso}

Esta unidad incluye rocas epiclásticas varicolores, todas de ambiente no marino. Se caracteriza por presentar en forma alternada secciones potentes de areniscas rojas, con otras de pelitas abigarradas bandeadas, bancos de yeso y rocas carbonáticas, cuyos afloramientos aparecen muy bien expuestos en la faja aflorante próxima a la Ruta Nacional 40. La recurrencia de facies y la falta de unanimidad en cuanto a la taxonomía estratigráfica de esta entidad entre los diferentes autores que trabajaron en la cuenca, parecen justificar el empleo de unidades estratigráficas informales en la subdivisión del mencionado grupo. En esta entidad se reconocen 11 secciones litológicas designadas con las letras C a M (Figura 3; Musacchio \& Vallati, 2000). Precisamente en la Sección K del Grupo H-R se registra la primera asociación con granos de angiospermas en la Zona de Afropollis zonatus (Vallati, 2001b, 2002c).

En el episodio regresivo del Grupo H-R en la Comarca Bajada del Agrio, tanto la litología como la micropaleontología (microfósiles calcáreos y palinomorfos) sugieren un episodio de continentalización con cuatro ciclos que muestran una tendencia no-lineal y repetición cíclica de litofacies (Musacchio \& Vallati, 2000; Figura 4). Esta transición del ambiente marino (niveles con amonites en el tope de la Formación Agrio) al ambiente continental parece estar relacionada con el descenso del nivel eustático global y con los movimientos epeirogénicos de reactivación tectónica (uplift) denominados movimientos miránicos iniciales (Stipanicic \& Rodrigo, 1969). La ausencia de sedimentación clástica gruesa en las litofacies de areniscas rojas (Secciones
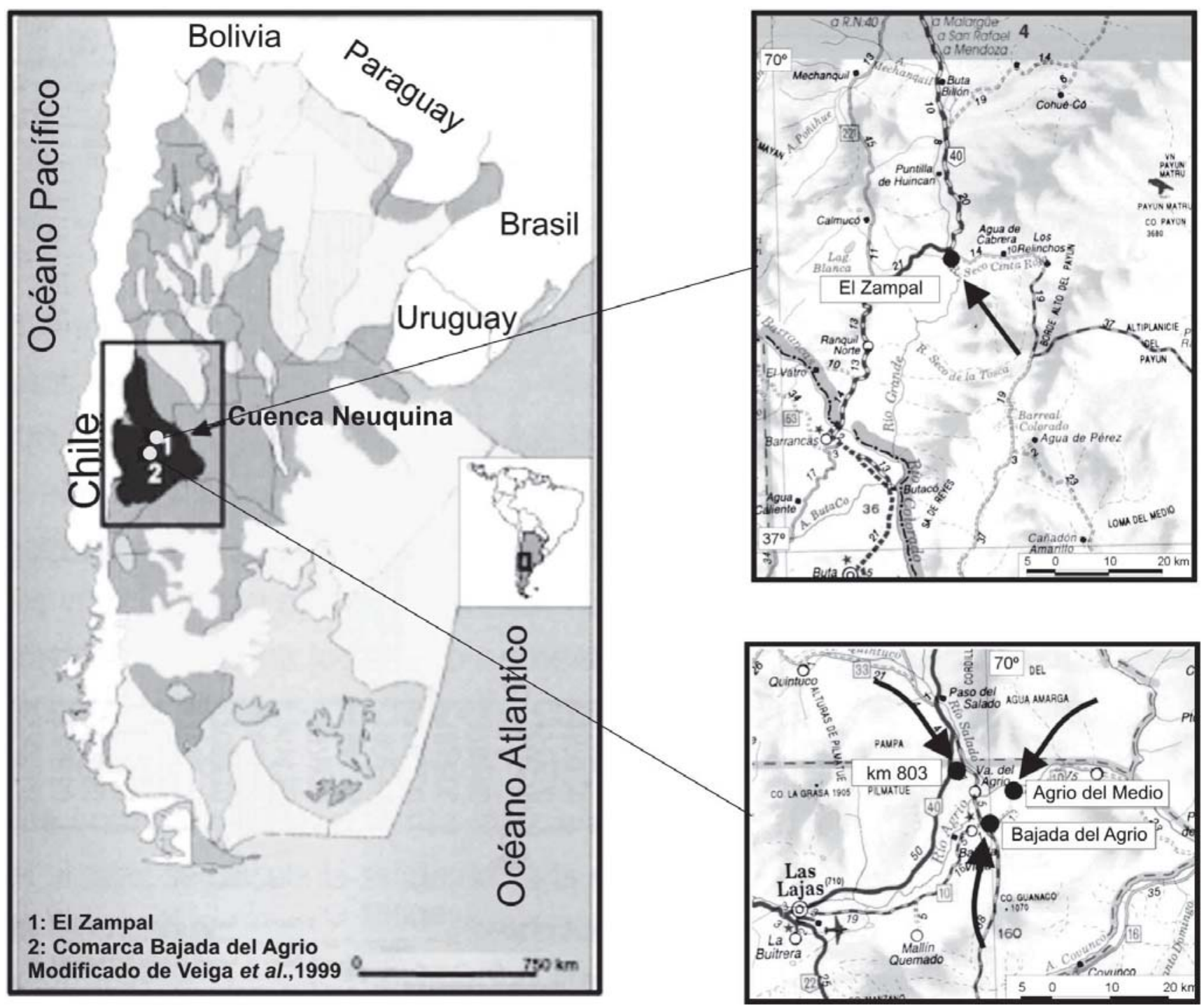

Figura 1. Mapa de ubicación del area estudiada.

Figure 1. Location map of the studied area. 


\section{Unidades estratigráficas mayores Movimientos tectónicos con amplia distribución}

d. Cubierta post-Daniana

Orogenia Quichua. Eoceno

Grupo Malargüe $\quad$ Movimientos Larámicos. Maastrichtiano-Daniano

c. "Riográndico"

Grupo Pehuenche (="Estratos con Dinosaurios")

m Orogenia Miránica principal. Cenomaniano?

\section{Grupo Huitrín-Rayoso}

m Rejuvenecimiento (uplift) Barremiano/Aptiano inferior regresión del Barremiano

\section{b. "Andico" Grupo Mendoza}

Form. Tordillo

"Loteniano-Chacayano"

\section{〜 m Pulso Catán Lil}

Orogenia Intermálmica. Oxfordiano

-transgresión del Caloviano

\section{a. "Jurásico"}

\section{"Cuyano"} Rebajamiento Gondwánico póstumo. Triásico

\section{"Basamento" complejo (Precámbrico, Paleozoico, Triásico)}

Figura 2. Esquema estratigráfico simplificado de la Cuenca Neuquina (Vallati, 2001b). Figure 2. Simplified stratigraphic scheme of the Neuquén Basin (Vallati, 2001b).

I, L, M en la Figura 3) sugiere que el ascenso continental responde a un abovedamiento amplio, antes que a la elevación de dorsales o fajas orogénicas. La repetición de facies de pelitas con foraminíferos y palinofloras que son paleoecológicamente comparables (Secciones K, G), sugiere la recurrencia de las condiciones ligadas a un contacto con el mar (Musacchio \& Vallati, 2000).

\section{"Estratos con Dinosaurios"}

Estos depósitos están constituídos por diferentes unidades de rocas epiclásticas varicolores. Los mismos representan la "molasa" post-orogénica que sigue a la Orogenia Miránica principal (también conocida como Orogenia Patagonídica principal). Estos movimientos son responsables de la discordancia angular (con manifestación en superficie y en el subsuelo) que separa los "Estratos con Dinosaurios" del Grupo H-R y del cambio de la vertiente pacífica de la cuenca (J-K1) a atlántica (K2?-K3).

Las formaciones Huincul y Cerro Lisandro han aportado asociaciones polínicas con granos tricolpados, tricolporoidados y tricolporados que representan un estadío relativamente más avanzado en la participación de las angiospermas (Zona de Fraxinoipollenites fragilis y Nivel con Cretacaeiporites polygonalis) (Vallati, 2001b, 2002c).

En Neuquén, la edad de los movimientos Patagonídicos principales está mal conocida. Pueden ser acotados, no obstante, entre el Aptiano (límite inferior máximo, correspondiente a la asociación con Afropollis) y el Cenomaniano (límite superior máximo, correspondiente a la palinoflora con granos triaperturados). Estos límites surgen, respectivamente, de las edades que el registro palinológico asigna a las secciones del Grupo H-R (Vallati, 1995, 1996a) y a las formaciones Huincul y Cerro Lisandro en Mendoza (Vallati, 2001a, 2002a).

\section{DATOS BIOESTRATIGRÁFICOS}

En el Cretácico medio de la Cuenca Neuquina se reconocieron las siguientes entidades bioestratigráficas (Vallati, 2001b, 2002c):

Zona de asociación Foraminisporis cf. variornatus 


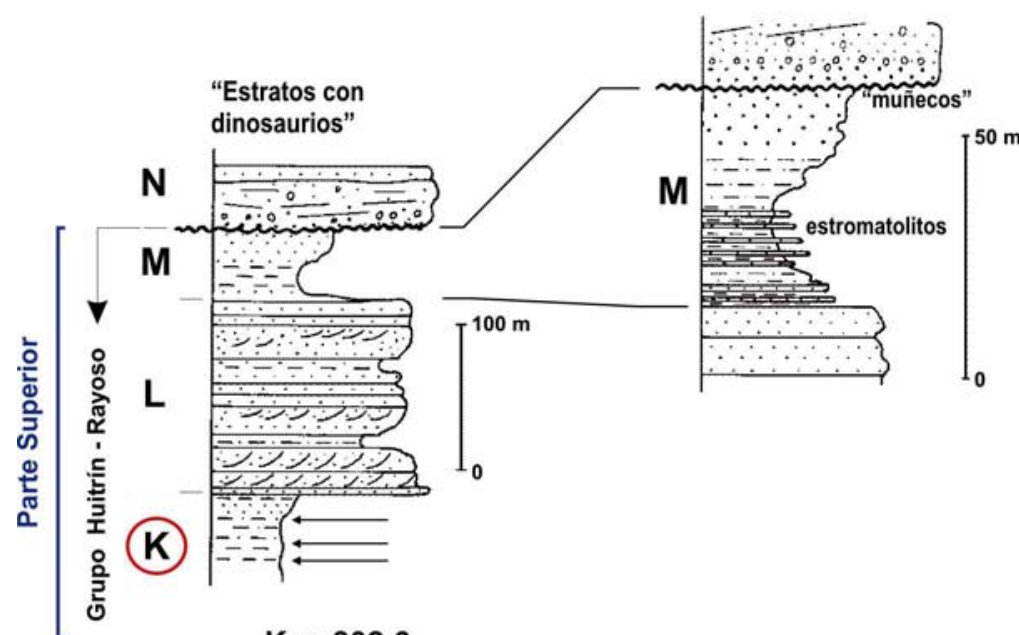

Km. 802.6
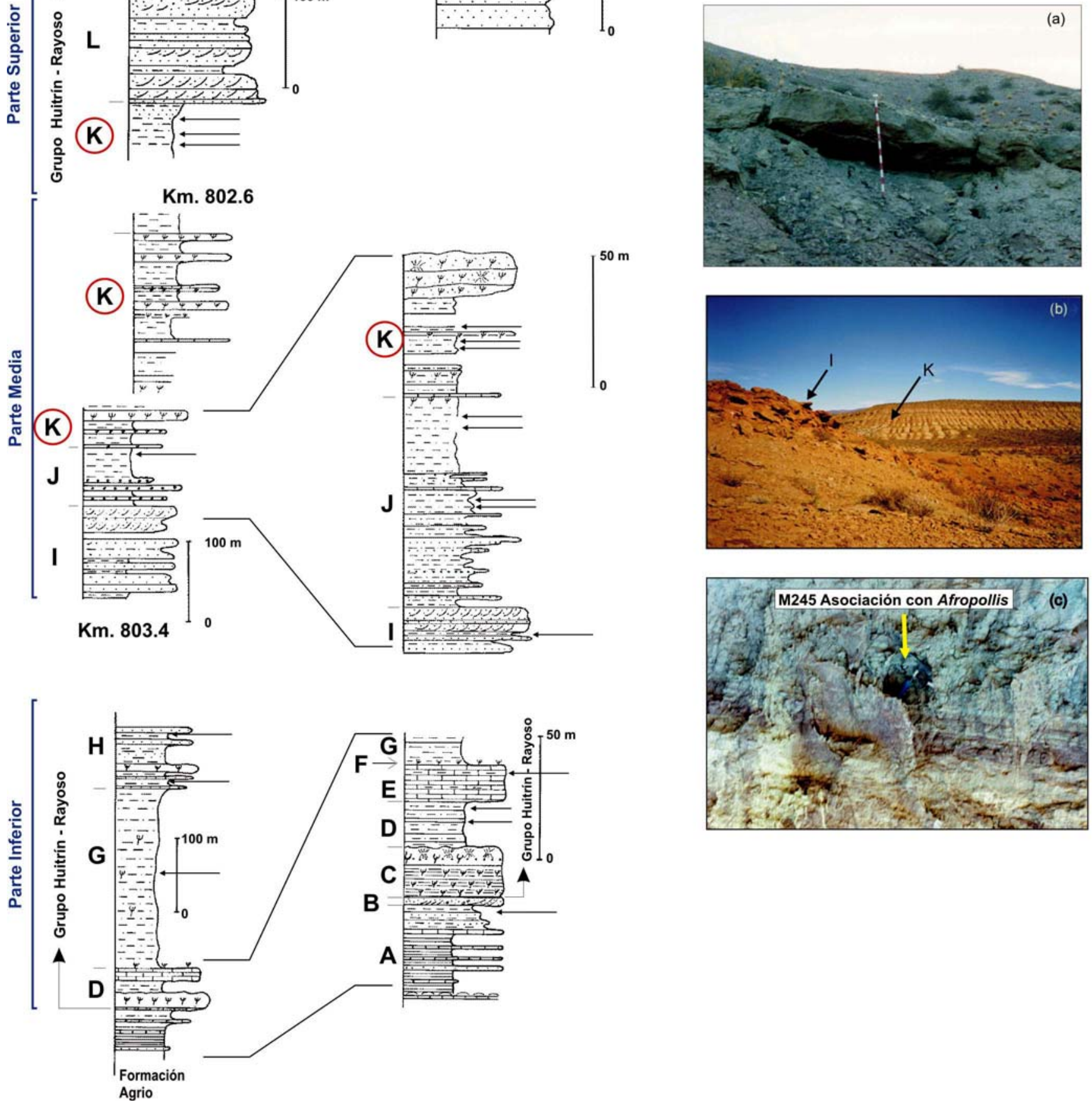

Figura 3. Perfil compuesto del Grupo Huitrín-Rayoso indicando los principales niveles fértiles (flechas). Secciones litológicas: A-N, ver Musacchio \& Vallati (2000); K, sección litológica con el primer registro de granos de angiospermas. (a) transición entre las secciones J y K; (b) vista de las areniscas rojizas de la Sección I y de las pelitas de la Sección K; (c) detalle de un sector en el afloramiento de la Sección K. Figure 3. Composite section of the Huitrín-Rayoso Group indicating the main fertile levels (arrows). Lithologic sections: A-N, see Musacchio \& Vallati (2000); K, lithologic section with the first record of angiosperm pollen grains. (a) transition between sections $\mathrm{J}$ and K; (b) View of the reddish sandstones of the Section $\mathrm{J}$ and the siltstones of the Section K; (c) detailed view of a sector in the Section $\mathrm{K}$ outcrop. 
(Barremiano tardío)

Subzona de Klukisporites foveolatus (Barremiano tardío)

Zona de asociación Afropollis zonatus (Aptiano)

Zona de asociación Fraxinoipollenites fragilis (Albiano?/ Cenomaniano)

Nivel con Cretacaeiporites polygonalis (Cenomaniano)

Los cambios principales en la participación de las angiospermas se verifican en las Zonas de Afropollis zonatus y Fraxinoipollenites fragilis con el primer registro y posterior diversificación y abundancia de las angiospermas respectivamente.

Las asociaciones palinológicas del Barremiano tardío en el Grupo Huitrín-Rayoso incluyen granos de gimnospermas dominantes y esporas de afinidad hepática junto con esporas de Lycophyta y Pteridophyta. La Subzona de Klukisporites foveolatus reconocida en las intercalaciones de fangolitas gris-azuladas de la sección $\mathrm{J}$ en la localidad de Bajada del Agrio, incluye más de 60 taxa de palinomorfos con el primer registro de varias especies de Gnetophyta (Singhia montanaensis, Gnetaceaepollenites sp., Gnetaceaepollenites barghoornii, Equisetosporites cf. albertensis) y de Triporoletes reticulatus. Los palinomorfos acuáticos están principalmente representados por algas prasinofíceas y quistes de acritarcos (Membranosphaera? sp.). No hay registro de granos de angiospermas en estas entidades bioestratigráficas basales del Grupo H-R.

Por su parte, la Zona de Afropollis zonatus (Aptiano) se caracteriza por presentar una asociación que incluye, hasta el momento, el primer registro de angiospermas primitivas en la comarca Bajada del Agrio (Figura 5). Afropollis está presente con varias especies, entre ellas Afropollis operculatus, Afropollis zonatus y Afropollis aff. jardinus. Además, hacen su aparición Clavatipollenites hughesii, Asteropollis asteroides, Asteropollis (Huitrinipollenites) transitorius, Retimonocolpites sp. y Liliacidites? sp. Otros taxa que caracterizan esta entidad son: Leptolepidites macroverrucosus, Pilosisporites trichopapillosus, Leptolepidites irregularis, Appendicisporites tricornitatus, Classopollis classoides, Cyclusphaera psilata, Balmeiopsis limbatus y Gnetaceaepollenites barghoornii. Entre los palinomorfos acuáticos, las algas prasinofíceas están muy bien representadas en algunos niveles.

Por su interés bioestratigráfico y biogeográfico se destaca la presencia de Afropollis, un género conspicuo de la Región paleoflorística Ecuatorial (Herngreen et al., 1996). Este género representa el $75 \%$ de las angiospermas primitivas presentes en la asociación y el 6\% del espectro polínico total (Vallati, 2001b).

La Sección tipo es la Sección K del Grupo Huitrín-Rayoso en el Perfil del Km. 803, en inmediaciones de la vieja Ruta Nacional 40, unos pocos kilómetros al norte del puente sobre el Río Agrio, en la Comarca Bajada del Agrio. Esta asociación también se reconoce en el perfil de Agrio del Medio, de la misma comarca. Asimismo, la asociación con Afropollis está presente en la localidad de Balsa Huitrín, de la misma cuenca.

En distintos niveles de la Formación Huincul, en la localidad de El Zampal, sur de Mendoza, se reconoce la Zona de asociación Fraxinoipollenites fragilis (Albiano?/ Cenomaniano). La misma incluye granos de angiospermas basales (Retimonocolpites, Clavatipollenites) junto a granos de eudicotiledóneas como Fraxinoipollenites fragilis, Psilatricolpites sp., Gemmatricolpites sp., Verrutricolpites sp., Tricolporoidites sp. (Figura 5B-G). Otros palinomorfos presentes son Cyclusphaera psilata, Balmeiopsis limbatus, Taurocusporites segmentatus, Triporoletes reticulatus. En la base de la Formación Huincul se ha reconocido Elateroplicites africaensis (Figura 6H). Este es el primer registro conocido para el Cretácico de Argentina de granos con elaterios. Estos últimos caracterizan la provincia paleoflorística ecuatorial en el Albiano-Cenomaniano (Herngreen \& Chlonova, 1981; Herngreen et al., 1996).

Por último, el Nivel con Cretacaeiporites polygonalis (Cenomaniano) se reconoce en la Formación Cerro Lisandro, que suprayace a la Formación Huincul en los "Estratos con Dinosaurios" basales (Figura 6I-P). Esta unidad, estudiada en la localidad de El Zampal, incluye una asociación con granos de eudicotiledóneas comparable a la presente en la Formación Huincul y está caracterizada por la primera aparición de Cretacaeiporites polygonalis, otro taxón característico de las provincias florísticas del Cretácico medio de Brasil (Herngreen, 1974a,b).

\section{Repositorio del material}

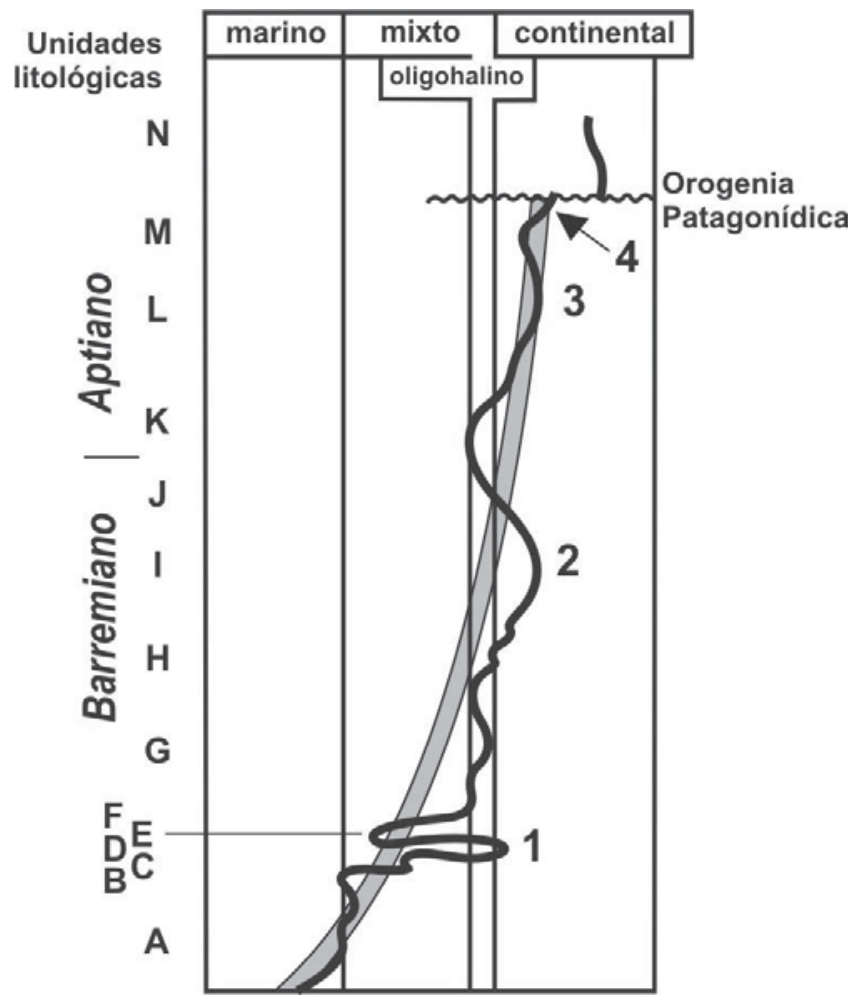

Figura 4. Curva de continentalización. Tendencia regresiva del Ciclo Ándico (en gris). 1-4: "Pulsos" de continentalización durante el intervalo Barremiano-Aptiano.

Figure 4. Cyclicity Design. Regresive trend of the Ándico Cycle (in grey). 1-4: terrestrial "pulses" during the Barremian-Aptian interval. 

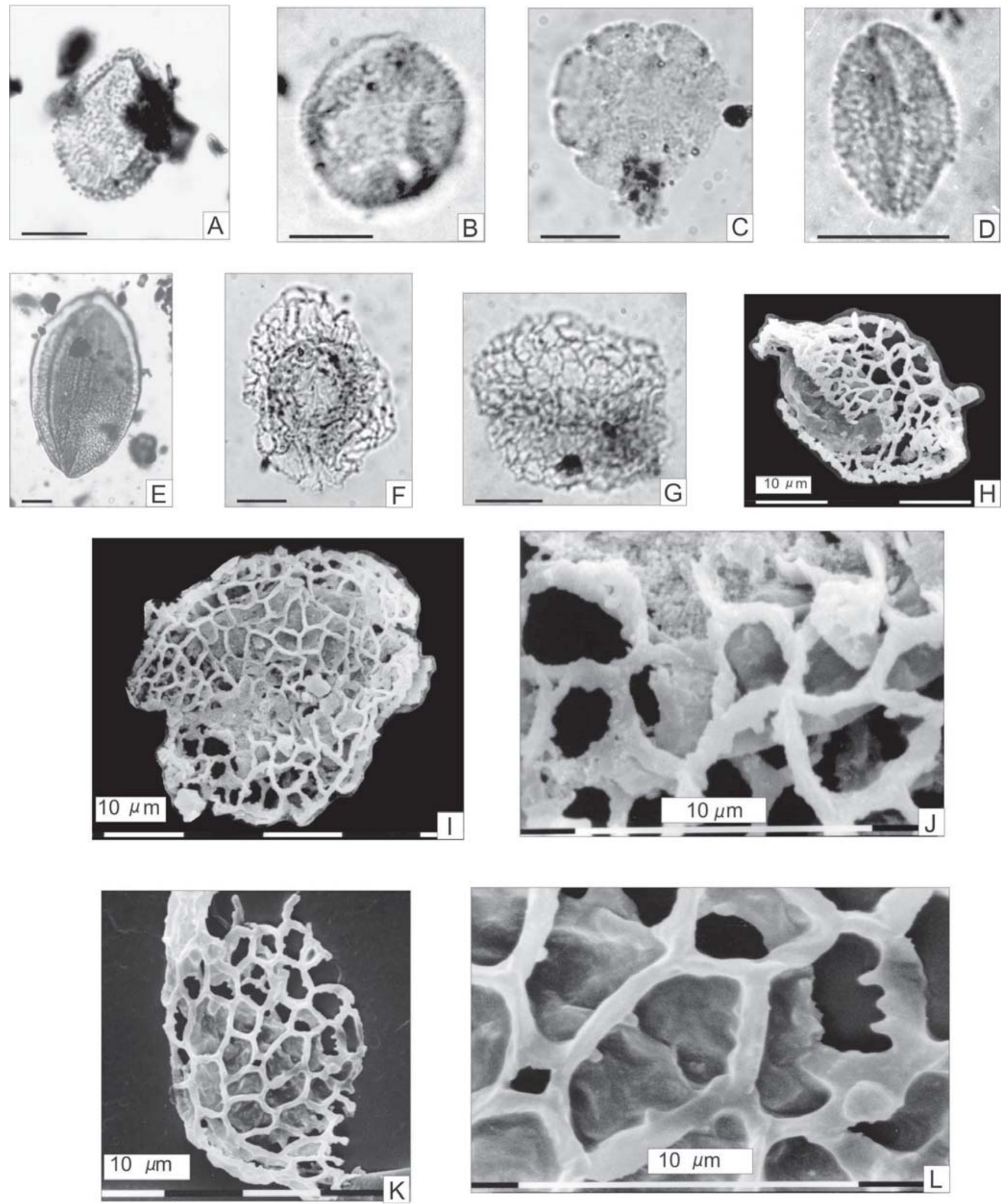

Figura 5. Zona de Afropollis zonatus, Aptiano de la Cuenca Neuquina (en paréntesis están los números de los preparados y las coordenadas): A, Clavatipollenites hughesii (CR.P.CV 80c1 3/101); B, Asteropollis asteroides (CR.P.CV 3752a 12/97); C, Asteropollis (Huitrinipollenites) transitorius (CR.P.CV 3753a 10/101); D, Retimonocolpites sp. (CR.P.CV 3751b 9/103); E, Liliacidites? sp. (CR.P.CV 80c1 17.5/95); F, Afropollis operculatus (CR.P.CV 249b 14/96); G, Afropollis zonatus (CR.P.CV 375R 2/95); H, Afropollis aff. zonatus (CR.P.CV MEB 0149/09); I, Afropollis zonatus (CR.P.CV MEB 143/09); J, Detalle del ejemplar ilustrado en I (Foto MEB); K, Afropollis cf. operculatus (CR.P.CV MEB 0141/09); L, Detalle del ejemplar ilustrado en K (Foto MEB). Escalas gráficas $=10 \mu \mathrm{m}$.

Figure 5. Afropollis zonatus Zone, Aptian of the Neuquén Basin (in parenthesis are the slide number and the coordinates). Scale bars = $10 \mu \mathrm{m}$. 

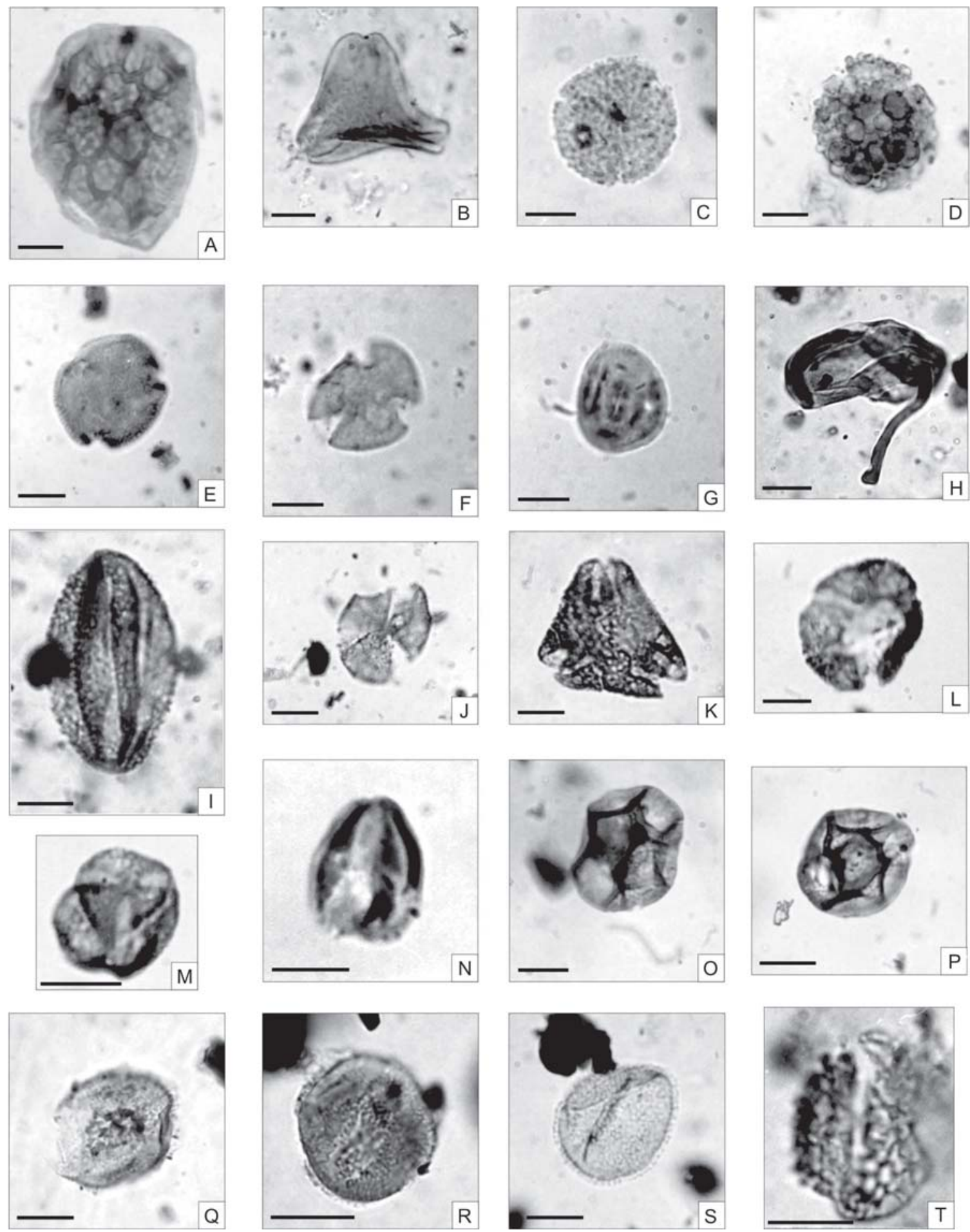

Figura 6. Zona de Fraxinoipollenites fragilis, Albiano?/Cenomaniano de la Cuenca Neuquina (en paréntesis están los números de los preparados y la coordinadas en el equipo citado): A, Triporoletes reticulatus (CR.P.CV 241x 15/92.5); B, Psilatricolpites sp. (CR.P.CV 241h 10/84); C, Verrutricolpites sp. (CR.P.CV 241h 5/99); D, Gemmatricolpites sp. (CR.P.CV 241h 3/97); E, Tricolporoidites sp. (CR.P.CV 241b 14/98); F, Fraxinoipollenites fragilis (CR.P.CV 241a 4/89); G, Tricolporoidites sp. (CR.P.CV 241b 3.4/86); H, Elateroplicites africaensis (CR.P.CV 536a 19.2/110). Nivel con Cretacaeiporites polygonalis (Cenomaniano de la Cuenca Neuquina): I, Fraxinoipollenites sp. (CR.P.CV 8632 20/99); J, Fraxinoipollenites cf. fragilis (CR.P.CV 863a 6/96); K, Tricolpites sp. (CR.P.CV 863x 12/114); L, Verrutricolpites? sp. (CR.P.CV 863R1 15/104); M, Tricolpites cf. micromunus (CR.P.CV 863Q2 4/98.5); N, Dryadopollis cf. argus (CR.P.CV 863R 7/87.2); O, Cretacaeiporites polygonalis (CR.P.CV 863a 10/108.5); P, Cretacaeiporites polygonalis (CR.P.CV 863t 17/90). Zona de Clavatipollenites hughesii (Barremiano tardío?/Aptiano temprano de la Cuenca del Golfo San Jorge): Q, Tucanopollis crisopolensis (CR.P.CV CCh4b 2/96); R, Tucanopollis cf. T. crisopolensis (CR.P.CV CCh4c 8.5/85.7); S, Clavatipollenites hughesii (CR.P.CV CCh4a 16/85); T, Retimonocolpites sp. (CR.P.CV CCh4d 22/102). Escalas gráficas $=10 \mu \mathrm{m}$.

Figure 6. Fraxinoipollenites fragilis Zone, Albian?/Cenomanian of the Neuquén Basin (in parenthesis are the slide number and the coordinates in the cited microscopy): A-H; Cretacaeiporites polygonalis Level, Cenomanian of the Neuquén Basin: I-P; Clavatipollenites hughesii Zone, late Barremian?/early Aptian of the San Jorge Gulf Basin: Q-T. Scale bars $=10 \mu \mathrm{m}$. 
Los preparados estudiados se encuentran archivados en las colecciones del Laboratorio de Bioestratigrafía de la Universidad Nacional de la Patagonia San Juan Bosco, bajo la sigla CR.P.CV. Las coordenadas que identifican los especimenes ilustrados corresponden a la escala Vernier del microscopio Carl Zeiss. La lista de las especies de palinomorfos mencionadas en el texto y/o ilustradas constituye el Apéndice.

\section{DISCUSIÓN}

En palinofloras incluídas en sedimentos cretácicos no marinos de la Cuenca Neuquina se reconocen dos saltos en la participación de las angiospermas: la primera aparición de granos representativos de este grupo vegetal (Zona de Afropollis zonatus), y el primer registro de eudicotiledóneas (Zona de Fraxinoipollenites fragilis). En los niveles estudiados no se ha encontrado aún el registro de una asociación de granos monosulcados tectados-columelados que están representados en el Barremiano de distintas partes del mundo, incluso en la Formación D-129 en Patagonia central (Vallati, 1996b). En efecto la Zona de Clavatipollenites hughesii (Vallati, 2001b, 2002c) reconocida en Sierra Silva, Chubut central, incluye las especies de angiospermas Clavatipollenites hughesii, Retimonocolpites sp. y Tucanopollis crisopolensis (Figura 6Q-T). Esta última especie, reconocida hasta ahora sólo en la Región Ecuatorial (Brasil y África), representa uno de los registros de angiospermas más antiguos para la mencionada región. Los niveles del Grupo H-R que infrayacen la asociación con Afropollis (Secciones litológicas D, G, I, J) incluyen asociaciones palinológicas en las que no se han reconocido granos de polen de angiospermas basales ( $i$ "hiatus" bioestratigráfico?). Asimismo, está faltando en la sucesión estudiada una asociación de granos tricolpados que represente a las primeras eudicotiledóneas. El posible hallazgo de niveles fértiles (no encontrados por el momento) en la parte superior del Grupo H-R y en la Formación Candeleros podría aportar evidencias de interés para interpretar la progresión de los tipos polínicos de angiospermas en el área estudiada.

En esta contribución se sugiere la posible vinculación de estas asociaciones que representan diferentes etapas en la participación de las angiospermas con los cambios fisiográficos asociados a la actividad tectónica. El primer evento palinoflorístico al que se hace referencia (el registro de los primeros granos de angiospermas), junto con el posible "hiatus" bioestratigráfico basal se relaciona con los movimientos miránicos iniciales, y la consecuente reactivación tectónica. El combamiento cortical (uplift) ha permitido la rotura del aislamiento geográfico que afectó a la biota en el Cretácico Inferior. Esto parece relacionarse con la presencia en la Cuenca Neuquina de taxa propios de la Región paleoflorística Ecuatorial (Afropollis en el Aptiano y Cretacaeiporites y Elateroplicites en el Cenomaniano), y probablemente también con el registro de Tucanopollis crisopolensis en el Barremiano tardío?/Aptiano temprano de la Cuenca del Golfo San Jorge. La presencia en Neuquén de estos elementos tropicales, junto a otras características presentes en las asociaciones neuquinas, han sugerido la ampliación de la extensión de la Provincia Transicional de Herngreen et al., 1996 (Vallati, 2002b).

Por su parte, la Orogenia Miránica principal fue responsable del cambio de pendiente regional de pacífica a atlántica. Se sugiere que la aparición de una dorsal protoandina en el sector occidental sudamericano ocasionó cambios en la fisiografía y en las condiciones climáticas imperantes, principalmente en el régimen de precipitaciones. La mencionada dorsal probablemente se comportó como una barrera para los vientos cargados de humedad, generando condiciones comparables a las existentes en la actualidad: bosques andinos húmedos $\mathrm{y}$ aridez creciente hacia el margen atlántico en la región extra-andina. Esto dio lugar al desarrollo de regiones húmedas, no sólo en el sector proto-andino, sino también en la región extra-andina inmediata (Cuenca Neuquina). Así, mientras las asociaciones del BarremianoAptiano del Gupo H-R muestran un dominio muy marcado de granos de Classopollis (más del 70\% en algunas palinofloras), que es un indicador aceptado de aridez ambiental, la palinología de las unidades basales de los "Estratos con Dinosaurios" evidencian un probable cambio climático (ausencia casi total de Classopollis y granos de angiospermas que dominan en un $60 \%$ la asociación). Las nuevas condiciones ambientales (aumento en las precipitaciones), probablemente fueron favorables para el desarrollo y la rápida diversificación de las eudicotiledóneas. Parece interesante señalar que en estos mismos niveles basales de los "Estratos con Dinosaurios" se ha reportado la presencia de abundantes troncos fosilizados y dinosaurios de gran tamaño (Bonaparte \& Coria, 1993).

Otros autores han vinculado los cambios paleoflorísticos con eventos tectónicos. En particular, para la Patagonia argentina, Archangelsky $(2001,2003)$ relaciona los cambios registrados en la distribución de las floras cretácicas (principalmente eventos de extinción en los grupos de Bennettittales y Ginkgoales) con la actividad volcánica en el Grupo Baqueró (Provincia de Santa Cruz).

\section{AGRADECIMIENTOS}

Mi sincero reconocimiento a Eduardo A. Musacchio por la valiosa discusión de los tópicos de esta contribución y la lectura crítica del manuscrito. Agradezco también a Cecilia Cunha Lana y Rodolfo Dino por los apreciados comentarios y sugerencias aportados en calidad de revisores del trabajo. Hago extensivo mi agradecimiento a Soraia Bauermann, por las oportunas correcciones al manuscrito. Finalmente, a Esteban Musacchio por su apreciada colaboración en el armado de las figuras de esta contribución.

\section{REFERENCIAS}

Aguirre Urreta, M.B. \& Rawson, P.F. 1993. The Lower Cretaceous ammonite Paraspiticeras from the Neuquén Basin, West Cen- 
tral Argentina. Neues Jahrbuch für Geologie und Paläontologie, Abhandlungen, 188(1):51-69.

Archangelsky, S. 2001. Evidences on an Early Cretaceous floristic change in Patagonia, Argentina. In: INTERNATIONAL SYMPOSIUM ON MESOZOIC TERRESTRIAL ECOSYSTEMS, 7, 1999. Special Publication, Buenos Aires, p. 15-19.

Archangelsky, S. 2003. The Ticó Flora (Patagonia) and the Aptian Extinction Event. Acta Palaeobotanica, 41(2):115-122.

Bonaparte, J.F. \& Coria, R.A. 1993. Un nuevo y gigantesco saurópodo titanosaurio de la Formación Río Limay (AlbianoCenomaniano) de la provincia del Neuquén, Argentina. Ameghiniana, 30(3):271-282.

Herngreen, G.F.W. 1974a. Palynology of Albian-Cenomanian strata of borehole 1-QS-1-MA, State of Maranhão, Brazil. Pollen et Spores, 15(3-4):514-555.

Herngreen, G.F.W. 1974b. Middle Cretaceous palynomorphs from northeastern Brazil. Bulletin Sciences Géologiques, 27(1/2):101-116.

Herngreen, G.F.W. \& Chlonova, A.F. 1981. Cretaceous microfloral provinces. Pollen et Spores, 23(3/4):441-555.

Herngreen, G.F.W.; Kedves, M.; Rovnina, L.V. \& Smirnova, S.B. 1996. Cretaceous Palynofloral Provinces: a review. In: J. Jansonius \& D.C. McGregor (eds.) Palynology: principles and applications, American Association of Stratigraphic Palynologists Foundation, 3:1157-1188.

Musacchio, E. \& Vallati, P. 2000. La regresión del BarremianoAptiano en Bajada del Agrio, Neuquén (Argentina). In: CONGRESO GEOLÓGICO CHILENO, 9, 2000. Actas, Puerto Varas, 2:230-234.

Stipanicic, P.N. \& Rodrigo, F. 1969. El diastrofismo Eo y mesocretácico en Argentina y Chile, con referencias a los movimientos jurásicos de la Patagonia. In: JORNADAS GEOLÓGICAS ARGENTINAS, 4, 1969. Actas, Mendoza, 2:337-352.

Vallati, P. 1995. Presencia de Afropollis (Polen de Angiosperma) en el Cretácico Inferior de la Cuenca Neuquina. In: CONGRESO ARGENTINO DE PALEONTOLOGÍA Y BIOESTRATIGRAFÍA, 4, 1995. Actas, Trelew, p. 277-290.

Vallati, P. 1996a. Palinología de la "Arenisca" Rincón (Grupo Huitrín/Rayoso), Cretácico Inferior de la Cuenca Neuquina. In: CONGRESO GEOLÓGICO ARGENTINO, 13, 1996. Actas, Buenos Aires, 5:77-92.

Vallati, P. 1996b. Palynology of the D-129 Formation in the San Jorge Gulf Basin, Lower Cretaceous, Patagonia, Argentina. In: CONGRESSO BRASILEIRO DE GEOLOGIA, 39, 1996. Anais, Salvador, UFBA, 7:423-426.

Vallati, P. 2001a. Middle Cretaceous microflora from the Huincul Formation ("Dinosaurian Beds") in the Neuquén Basin, Patagonia, Argentina. Palynology, 25:179-197.

Vallati, P. 2001b. Bioestratigrafía (Palinología) del Cretácico temprano y medio en Patagonia septentrional y central. Universidad Nacional de la Patagonia San Juan Bosco, Tesis Doctoral, $278 \mathrm{p}$.

Vallati, P. 2002a. Palynology of the Cerro Lisandro Formation (lower "Dinosaurian Beds") in the Neuquén Basin, west-central Argentina. Neues Jarbuch für Geologie und Paläontologie, Abhandlungen, 224(3):411-428.

Vallati, P. 2002b. Asociaciones palinológicas en el Cretácico temprano y medio de Patagonia septentrional: algunas consideraciones paleobiogeográficas. In: SIMPOSIO SOBRE
EL CRETÁCICO DE AMÉRICA DEL SUR, 2, 2002. Resumos expandidos, São Pedro, UNESP, p. 157-161.

Vallati, P. 2002c. Biostratigraphy of Cretaceous non-marine sequences in Patagonia. In: EUROPEAN MEETING ON THE PALEONTOLOGY AND STRATIGRAPHY OF LATIN AMERICA, 3, 2002. Expanded Abstracts, Toulouse, p.124-127.

Veiga, R.; Lara, M.E. \& Bruveris, P. 1999. Distribución de hidrocarburos sobre el margen externo en una cuenca de trasarco. Ejemplos en la cuenca Neuquina, Argentina. Boletín de Informaciones Petroleras, 60:142-164.

Received in January, 2006; accepted in March, 2006.

Apéndice. Lista de especies de palinomorfos mencionadas en el texto y/o ilustradas.

Appendix. List of palynomorph species cited and/or illustrated in the text.

Esporas de Hepatophyta, Pteridophyta y de afinidad incierta

Appendicisporites tricornitatus Weyland \& Greifeld 1953

Foraminisporis cf. variornatus Archangelsky 1983

Klukisporites foveolatus Pocock 1965

Leptolepidites irregularis (Brenner) Wingate 1980

Leptolepidites macroverrucosus Schultz 1967

Pilosisporites trichopapillosus (Thiegart) Delcourt \& Sprumont 1955

Taurocusporites segmentatus Stover 1962

Triporoletes reticulatus (Pocock) Playford 1971 (Figura 6A)

Granos de polen de Gimnospermas

Balmeiopsis limbatus (Balme) Archangelsky 1977

Classopollis classoides (Pflug) Fensome 1983

Cyclusphaera psilata Volkheimer \& Sepúlveda 1976

Elateroplicites africaensis Herngreen 1973 (Figura 6H)

Equisetosporites cf. albertensis Singh 1964

Gnetaceaepollenites sp. (en Vallati 2001b)

Gnetaceaepollenites barghoornii (Pocock) Lima 1980

Singhia montanaensis (Brenner) Lima 1980

\section{Granos de polen de Angiospermas}

Afropollis aff. zonatus Doyle, Jardiné \& Doerenkamp 1982 (Figura $5 \mathrm{H})$

Afropollis operculatus Doyle, Jardiné \& Doerenkamp 1982 (Figura $5 \mathrm{~F})$

Afropollis cf. operculatus Doyle, Jardiné \& Doerenkamp 1982 (Figuras 5K,L)

Afropollis zonatus Doyle, Jardiné \& Doerenkamp 1982 (Figura 5G) Asteropollis asteroides Hedlund \& Norris 1968 (Figura 5B)

Asteropollis (Huitrinipollenites) transitorius (Volkheimer \& Salas) nov. comb. (Figura 5C)

Clavatipollenites hughesii (Couper) Kemp 1968 (Figuras 5A y 6S)

Cretacaeiporites polygonalis (Jardiné \& Magloire) Herngreen 1974 (Figuras 6O,P)

Dryadopollis cf. argus Srivastava 1975 (Figura 6N)

Fraxinoipollenites fragilis Burger 1993 (Figura 6F)

Fraxinoipollenites cf. fragilis Burger 1993 (Figura 6J)

Fraxinoipollenites sp. (en Vallati 2002a) (Figura 6I)

Gemmatricolpites sp. (en Vallati 2001a) (Figura 6D)

Liliacidites? sp. (en Vallati 2001b) (Figura 5E)

Psilatricolpites sp. (en Vallati 2001a) (Figura 6B)

Retimonocolpites sp. (en Vallati 1995) (Figura 5D)

Retimonocolpites sp. (en Vallati 1996b) (Figura 6T) 
Tricolpites cf. micromunus (Groot \& Penny) Burger 1970 (Figura $6 \mathrm{M})$

Tricolpites sp. (en Vallati 2002a) (Figura 6K)

Tricolporoidites sp. (en Vallati 2001a) (Figuras 6E,G)

Tucanopollis crisopolensis (Regali et al.) Regali 1989 (Figura 6Q)

Tucanopollis cf. T. crisopolensis (Regali et al.) Regali 1989

(Figura 6R)

Verrutricolpites sp. (en Vallati 2001a) (Figura 6C)

Verrutricolpites? sp. (en Vallati 2002a) (Figura 6L)

Quistes de Acritarcos

Membranosphaera? sp. (en Vallati 1995) 